\title{
Comparative proteomic analysis of the esophageal squamous carcinoma cell line EC109 and its multi-drug resistant subline EC109/CDDP
}

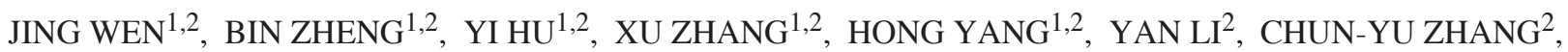 \\ KONG-JIA LUO ${ }^{1,2}$, XING ZANG $^{1,2}$, YONG-FENG LI ${ }^{1,2}$, XIN-YUAN GUAN ${ }^{2}$ and JIAN-HUA FU ${ }^{1,2}$ \\ ${ }^{1}$ Department of Thoracic Oncology, ${ }^{2}$ State Key Laboratory of Oncology in Southern China, \\ Cancer Center, Sun Yat-Sen University, Guangzhou 510060, P.R. China
}

Received July 27, 2009; Accepted September 21, 2009

DOI: 10.3892/ijo_00000497

\begin{abstract}
To gain insights into the mechanisms of drug resistance in esophageal squamous cell carcinoma (ESCC), we employed proteomic techniques to study the global protein change of the multi-drug resistant ESCC cell line EC109/CDDP, which was established in our previous work, in comparison with its parental drug sensitive cell line EC109. By two-dimensional electrophoresis and mass spectrometry, we successfully identified 44 proteins with altered expression levels. These proteins are involved in endoplasmic reticulum stress response, metabolic process, DNA replication and repair, nucleotide binding, calcium binding, and cytoskeletal proteins. Among them, the differential expression levels of thioredoxin domain-containing protein 4 precursor and cystathionine $\gamma$-lyase were further validated by Western blot and RT-PCR. Our present results lay foundation for future in-depth work on molecular mechanism of ESCC drug resistance, and aid in the identification and use of novel markers in clinical practice.
\end{abstract}

\section{Introduction}

Esophageal cancer (EC) is one of the most common malignancies in the world. It was estimated that $>300,000$ people die of it every year, ranking as the sixth leading cause of cancer death worldwide (1). Most esophageal cancers in China are squamous cell carcinomas (SCCs), which are different from the most prevalent esophageal cancers, adenocarcinomas, in Western countries, leading to distinct etiologies, therapeutics and prognosis (2). Despite advances

Correspondence to: Professor Jianhua Fu, Department of Thoracic Oncology, Cancer Center, Sun Yat-Sen University, State Key Laboratory of Oncology in Southern China, 651 Dongfeng East Road, Guangzhou 510060, P.R. China

E-mail: fu_jianhua@hotmail.com

Key words: esophageal squamous cell carcinoma, multidrug resistance, proteomics in surgical therapy for esophageal squamous cell carcinoma (ESCC), the overall prognosis of patients has not improved markedly during the past few decades due to the fact that most patients have locally-advanced or disseminated diseases at diagnosis (3). For these unresectable diseases, chemotherapy alone (stage IV) or in combination with other local treatment modalities (stages II and III) would be an optimal choice. A recent meta-analysis indicated that preoperative chemoradiotherapy with surgery would bring about $13 \%$ absolute difference in survival at 2 years versus surgery alone (4). However, the success of chemotherapy depends on the sensitivity of the tumor to the antineoplastic agents. ESCC cells often acquire resistance to drugs and even develop multiple drug resistance, which results in treatment failure. To achieve more effective chemotherapeutic treatment of ESCC patients, it is essential to define reliable indicators of response to treatment in individual patients and to make clear which mechanisms are responsible for drug resistance.

A lot of work has been done on the molecular mechanisms of ESCC drug resistance. It has been reported that factors including growth-factor receptor, angiogenetic factors, tumor suppressor genes, cell cycle regulators, and DNA repair enzymes are potential EC chemo-sensitivity predictive markers (5-11). The development of techniques within genomics and proteomics enables extensive characterization of malignant tumors, which may help in understanding treatment resistance and/or treatment sensitivity on the global scale. Gene expression studies on mRNA level have shown to be able to detect molecular signature linked to acquired resistance to cisplatin in ESCC cells (12). However, several aspects in tumor biology cannot be captured by gene expression analysis only, such as protein expression levels, protein degradation and post-translational modifications, emphasizing the need for complementary analysis at the protein level. Proteomic studies based on twodimensional electrophoresis (2-DE) have been adopted to study chemotherapy resistance of various tumor cell lines, including breast cancer, lung cancer, and colon cancer (13). However, there is no previous global protein analysis by 2-DE on ESCC cell lines of different drug sensitivity. We still lack protein markers that show direct relevance to ESCC chemoresistance. 
To gain new insights into the mechanisms of drug resistance in ESCC, we employed proteomic techniques to analyze protein extracts from the ESCC drug resistant cells EC109/CDDP (14) and its parental cells EC109 in the present study. After comparing the expression patterns, differentially expressed proteins were identified by mass spectrometry (MS) and further validated by Western blot and RT-PCR.

\section{Materials and methods}

Cell culture. Both EC109 and EC109/CDDP cells were cultured in Dulbecco's modified Eagle's medium (DMEM) (Invitrogen, NY, USA) supplemented with $10 \%$ fetal bovine serum, $100 \mathrm{U} / \mathrm{ml}$ penicillin (Tianxin, Guangzhou, China) and $100 \mathrm{U} / \mathrm{ml}$ streptomycin (Merro, Dalian, China) in a humidified incubator containing $5 \% \mathrm{CO}_{2}$ at $37^{\circ} \mathrm{C}$. The EC109/CDDP cells were generated by exposing EC109 cells to cisplatin at $2 \mathrm{~h}$ pulse treatment of $25 \mu \mathrm{M}$ for 6 times (14). Previous studies identified that EC109/CDDP cells, as compared with the parental EC109 cells, showed cross-resistance to cisplatin, carboplatin, 5-fluorouracil, taxol, navelbine, irinotecan and etoposide. Only EC109 and EC109/CDDP cells with the $\mathrm{IC}_{50}$ of cisplatin at $\sim 1.0 \mu \mathrm{g} / \mathrm{ml}$ and $8.5 \mu \mathrm{g} / \mathrm{ml}$ determined by MTT assay (14), respectively, were used for the following 2-DE, Western blot and RT-PCR analyses, in order to guarantee the homogeneity of the cells collected, at least, regarding their resistance to cisplatin.

Two-dimensional gel electrophoresis. EC109/CDDP cells and EC109 cells were harvested and dissolved in proper amount of lysis buffer containing $7 \mathrm{M}$ urea (Amersham Biosciences, Piscataway, NJ, USA), 2 M thiourea (Amersham Biosciences), 4\% (w/v) 3-[(3-cholamidopropyl) dimethylammonio]-1propanesulfonate (CHAPS) (Amersham Biosciences), $40 \mathrm{mM}$ 1,4-dithio-DL-threitol (DTT) (Amersham Biosciences) and a protease inhibitor cocktail tablet (Roche Applied Science, Mannheim, Germany). The protein concentration was determined by 2-D Quant Kit (Amersham Biosciences) and stored at $-80^{\circ} \mathrm{C}$.

The 2-DEs were carried out as described (15). Each precast immobilized $\mathrm{pH}$ gradient (IPG) strip ( $\mathrm{pH}$ 3-10 NL, $18 \mathrm{~cm}$ ) (Amersham Biosciences) was loaded with $50 \mu \mathrm{g}$ proteins diluted in $340 \mu 1$ rehydration solution containing $7 \mathrm{M}$ urea, $2 \mathrm{M}$ thiourea, 4\% CHAPS, $40 \mathrm{mM}$ DTT, $0.5 \%$ IPG buffer (pH 3-10, NL) (Amersham Biosciences) and a trace of bromophenol blue. Rehydration and IEF were performed in a Protean IEF cell apparatus (Bio-Rad, Hercules, CA, USA) using following conditions: (1) $50 \mathrm{~V}, 12 \mathrm{~h}$; (2) $250 \mathrm{~V}, 15 \mathrm{~min}$; (3) 10,000 V, $3 \mathrm{~h}$, linear; (4) 10,000 V, 50,000 VH. Then, the strips were equilibrated in the SDS equilibration buffer $(6 \mathrm{M}$ urea, $75 \mathrm{mM}$ Tris- $\mathrm{HCl} \mathrm{pH} 8.8,29.3 \%$ glycerol, $2 \%$ SDS and $0.002 \%$ bromophenol blue) containing $1 \%$ DTT for $15 \mathrm{~min}$ and then in another SDS equilibration buffer containing $2.5 \%$ iodoacetamide instead of DTT for additional $15 \mathrm{~min}$. For the second dimension, $12.5 \%$ SDS-PAGE were run in Protean II XL cell apparatus (Bio-Rad). For each sample, three replicate gels were run.

After electrophoresis, the protein gels were visualized by silver stain using PlusOne Silver Staining Kit (Amersham Biosciences) according to the manufacturer's instruction. The method was modified to be compatible with mass spectrometry analysis by omitting glutaraldehyde from the sensitizer solution.

Analysis of gel images. Silver-stained gels were scanned using a Molecular Imager GS-800 Calibrated Densitometer (Bio-Rad). Raw images from 2-DE gels were analyzed using the PDQuest 2-D Analysis Software (Version 7.4, Bio-Rad) according to the manual. The raw quantity of each spot in a gel was normalized by the total quantity of valid spots in that gel in order to accurately compare spot quantities between gels. The three replicate gels of each sample were defined as a replicate group. The spots displaying a change in their expression between the two groups greater than the two-fold factor (determined from the mean) significantly $(\mathrm{P}<0.05)$ were considered as differentially expressed proteins.

Mass spectrometric analysis of proteins. Silver-stained protein spots of interest were excised from the gels and digested with trypsin $(20 \mu \mathrm{g} / \mathrm{ml}$, Promega Corporation, Madison, WI, USA) according to manufacturer's instruction. Then the samples were analyzed in a positive ion reflector mode on a 4800 plus MALDI-TOF-TOF Analyzer Proteomic Analyzer (Applied Biosystems, Foster City, CA, USA). Five MS peaks of each sample with a signal-to-noise ratio above 50 were subjected to further MS/MS analysis. All MS and MS/MS data of each individual spot were analyzed by GPS Explore (Version 3.6, Applied Biosystems) and searched in International Protein Index (IPI)_human database searching through MASCOT (Version 2.1, Matrix Science, London, UK). Only identified proteins with a confidence interval (CI) of either protein or ion score $>95 \%$ were accepted.

Western blot. EC109 and EC109/CDDP cells were dissolved using 1xRIPA lysis buffer (Upstate, Lake Placid, NY, USA), respectively. Proteins were separated using 12\% SDS-PAGE and electrophoretically transferred onto polyvinylidene difluoride membranes (Amersham Biosciences). After blocked in Tris-buffered saline containing 5\% (w/v) non-fat dry milk and $0.1 \%(\mathrm{v} / \mathrm{v})$ Tween-20 for $1 \mathrm{~h}$ at room temperature, the membranes were then probed with following primary antibodies: rabbit polyclonal anti-thioredoxin domain-containing protein 4 (TXNDC4) antibody (Cell Signaling Technology, Beverly, MA, USA), mouse monoclonal anti-cystathionine $\gamma$-lyase $(\mathrm{CTH})$ antibody (Abnova, Jhouzih St., Taibei, Taiwan), and mouse monoclonal anti-ßactin antibody (Thermo Scientific, Fremont, CA, USA) overnight at $4^{\circ} \mathrm{C}$, followed by incubation with HRP-conjugated secondary antibodies, sheep anti-mouse IgG (Amersham Biosciences) or goat anti-rabbit IgG (Zymed, San Francisco, CA, USA) for $1 \mathrm{~h}$ at room temperature. Finally, the protein bands were visualized on the X-ray sensitive film using the enhanced chemiluminescence detection system (Amersham Biosciences). The specific intensity of each protein band on X-ray film was measured by Quantity One V4.62 (Bio-Rad) and expressed as a ratio of the optical density band of each protein to that of $\beta$-actin.

Semi-quantitative RT-PCR. Total RNA was isolated from EC109 and EC109/CDDP cells using TRIzol reagent 
Table I. Primers used for RT-PCR analysis.

\begin{tabular}{|c|c|c|c|c|}
\hline Gene & Primers $\left(5^{\prime}{ }^{\prime} 3^{\prime}\right)$ & Accession number & $\begin{array}{c}\text { Annealing } \\
\text { temperature }\left({ }^{\circ} \mathrm{C}\right)\end{array}$ & $\begin{array}{l}\text { Size } \\
\text { (bp) }\end{array}$ \\
\hline CTH 1 & & NM_001902 & 51 & 800 \\
\hline Sense & CCATCTCACTGTCCACCAC & & & \\
\hline Anti-sense & CTGACGCTTCACCAACTC & & & \\
\hline CTH 2 & & NM_153742 & 51 & 668 \\
\hline Sense & ССАТСТСАСТGТССАССАС & & & \\
\hline Anti-sense & CTGACGCTTCACCAACTC & & & \\
\hline TXNDC4 & & NM_015051 & 50 & 240 \\
\hline Sense & TTCCGATGTCATTAAGGAAG & & & \\
\hline Anti-sense & GCTAAGTCCCGAATTTCTTG & & & \\
\hline$\beta$-actin & & NM_001101 & 55 & 619 \\
\hline Sense & CGGGACCTGACTGACTACCTCATCAAGA & & & \\
\hline Anti-sense & TCAAGAAAGGGTGTAACGCAACTA & & & \\
\hline
\end{tabular}

(Invitrogen). RNA was reverse-transcripbed using the First Strand cDNA Synthesis Kit (Toyobo, Osaka, Japan). Then cDNA was amplified by PCR using rTaq DNA polymerase (Toyobo). The primers sequences, length of products, and PCR reaction conditions are listed in Table I. Agarose gels $(1.5 \%)$ were used to separate PCR products, and visualized by Gel Doc (Bio-Rad). The level of specific mRNA was measured by Quantity One V4.62 (Bio-Rad) and expressed as a ratio of the optical density band of each gene to that of the house-keeping gene, $\beta$-actin.

Statistical analysis. Data are expressed as mean \pm standard deviation (SD) of at least 3 repeated experiments. Data analysis was carried out utilizing the SPSS 16.0 statistical software package (SPSS, IL, USA). Continuous variables were analyzed using Student's t-test. $\mathrm{P}<0.05$ was considered statistically significant, and the reported P-values were twosided.

\section{Results}

Proteomic analysis of EC109 and EC109/CDDP cells. Proteomic profiles of EC109 and EC109/CDDP cells were investigated by 2-DE. Totally 1248 protein spots were observed, which were localized in the range of pI 3-10 with a relative molecular mass of $10-200 \mathrm{kDa}$. The analysis of the six 2-DE gels obtained for EC109 and EC109/CDDP cells with three replicas per sample showed that $88.7 \%$ $(1107 / 1248)$ and $84.6 \%(1053 / 1248)$ of the spots matched among gels of EC109 and EC109/CDDP cell protein sample, respectively (Fig. 1). The mean overall coefficients of variation (CVs) (SD/mean $\times 100 \%)$ of the matched spot quantities in EC109 and EC109/CDDP's replicate gels were 27.6 and $30.6 \%$, respectively, consistent with the conclusion drawn from previous reports that the average baseline technical variation from the 2-DE separation and detection process accounts for quantitative variation in the range of $20-30 \%$ (16). Furthermore, 27 spots were found more than two-fold up-regulated and 34 down-regulated in EC109/ CDDP cells as compared with EC109 cells by PDQuest analysis $(\mathrm{P}<0.05)$.

Protein identification. Among the 61 differentially expressed spots between EC109 and EC109/CDDP cells, 55 were cut from the gels for MS analysis and $44(83.6 \%)$ spots were identified with CI of either protein or ion score $>95 \%$ (Table II; Figs. 2 and 3). Six spots up-regulated in EC109 cells were not subjected to MS analysis, for they were too obscure or faint to cut from gels by visual inspection. Three spots upregulated in EC109 and 8 in EC109/CDDP cells, respectively, analyzed by MS and MS/MS analysis with the CI of either protein or ion score $<95 \%$ were not recorded as successfully identified.

Spot SSP 7117 was identified as two unique proteins. Likewise, a large number of spots were identified as proteins with isoforms or variants of one another, such as spots SSP $2117,6115,6305,8103$, and 8115 . This might be caused by the partial same amino acid sequence of different proteins or isoforms, which could not be distinguished by MS and MS/MS analysis precisely. Further experiments should be performed to confirm which protein or isoform affects the drug-resistance phenotype.

Some of the identified proteins migrated at PI and MW values different to the theoretical ones. Two spots SSP 0308 and 0309, both up-regulated in EC109 cells, were identified as the same protein reticulocalbin-1 precursor ( $\mathrm{RCN} 1$, IPI00015842). It might be due to either processing or posttranslational modification resulting in different positions in the gel for a given gene product. Characterization of these proteins in terms of modification type was not attempted in this study.

Western blot. The protein expression of two identified proteins up-regulated in EC109/CDDP cells of interest by 2-DE and MS, TXNDC4 (SSP 2338) and CTH (SSP 6305), were selected for further validation using Western blot. The 


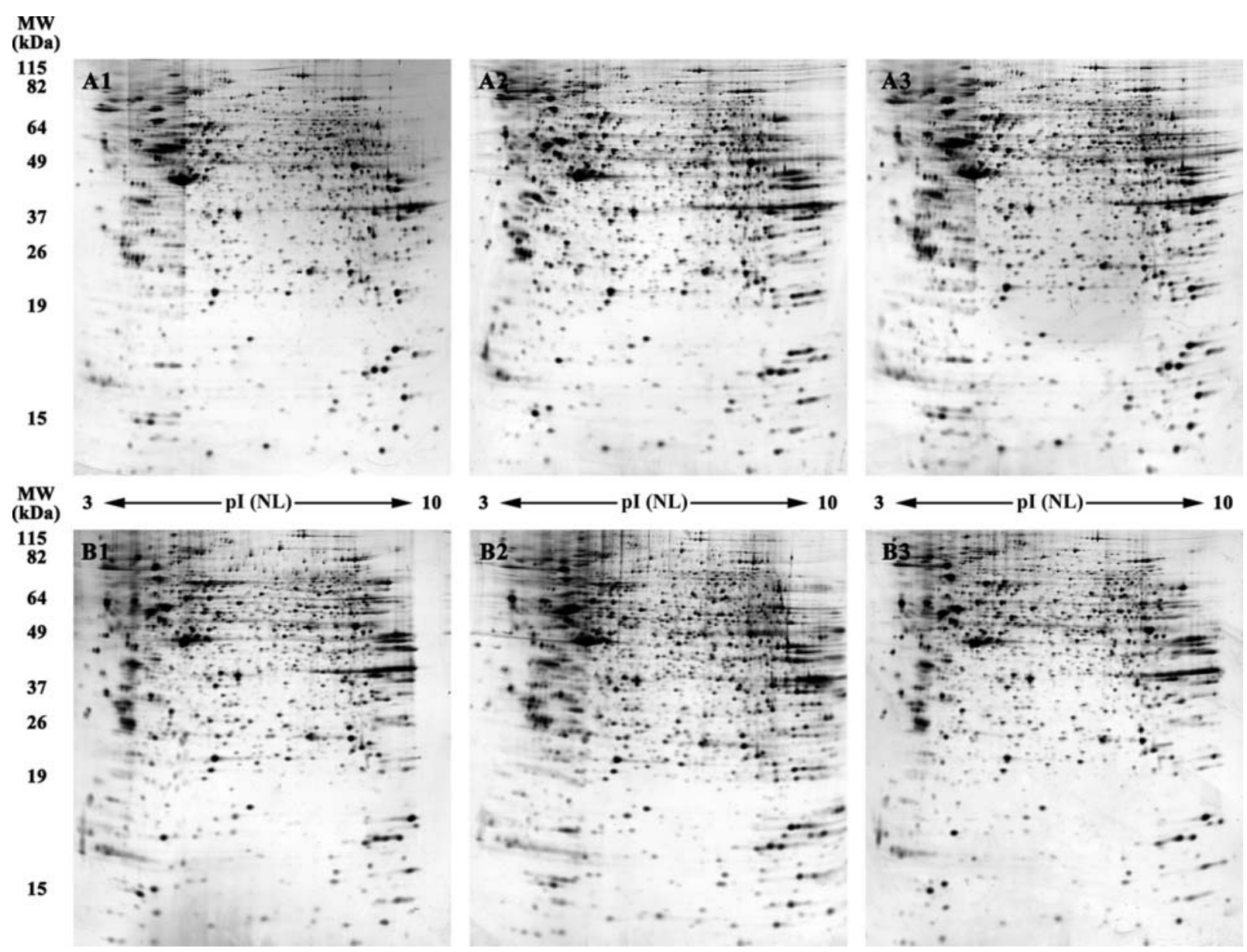

Figure 1. Three replicate 2-DE silver-stained gel images of EC109 (A1-A3) and EC109/CDDP (B1-B3) cell proteins, respectively.

\section{3}

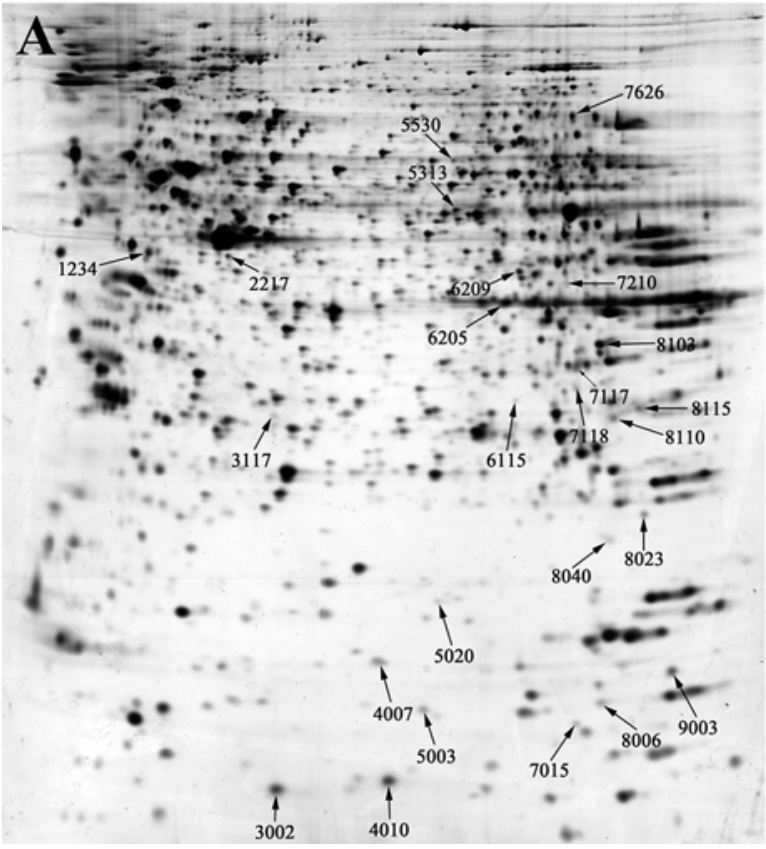

MW

(kDa)
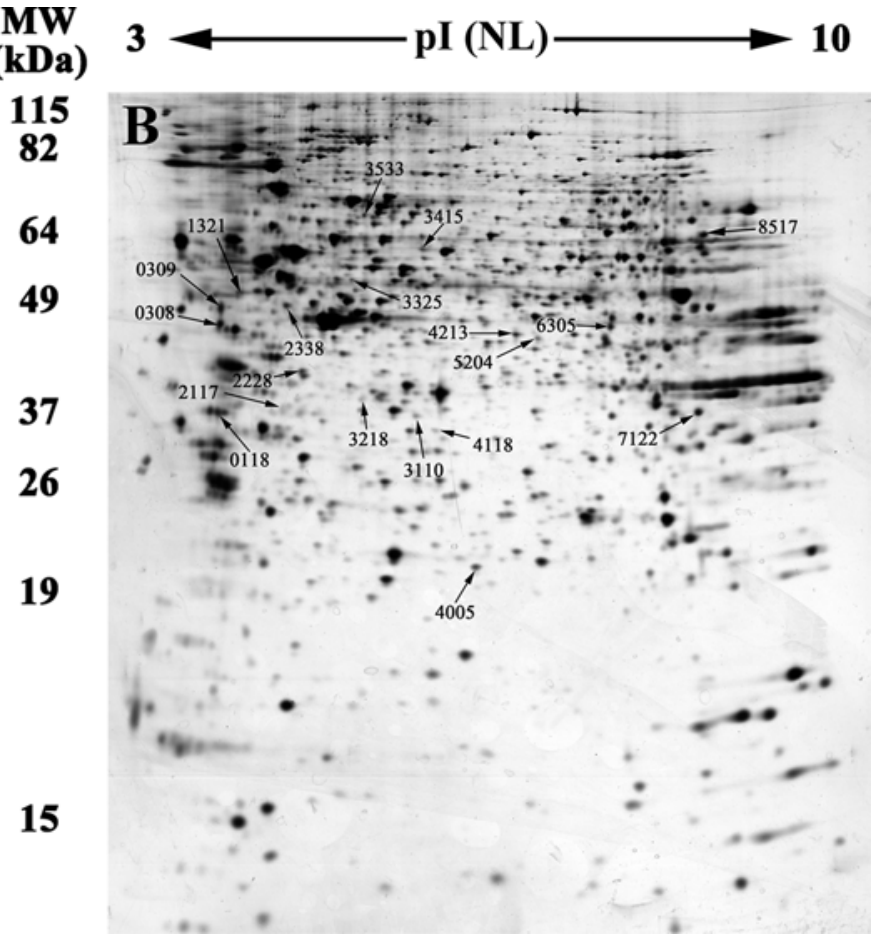

Figure 2. Representative 2-DE gels (pI 3-10, NL, $18 \mathrm{~cm}$ ) showing the location of 25 protein spots up-regulated in EC109 (A) cells and 19 in EC109/CDDP (B) with arrows. The number in the image is the SSP (standard spot) number assigned uniquely to each spot by PDQuest software. 
Table II. Identified increased and decreased proteins in drug-resistant cell line EC109/CDDP relative to parental EC109.

\begin{tabular}{|c|c|c|c|c|c|c|}
\hline $\begin{array}{l}\text { SSP } \\
\text { No. }\end{array}$ & Gene symbol & Protein name & Accession No. ${ }^{a}$ & $\begin{array}{c}\text { Fold } \\
\text { change }^{b}\end{array}$ & $\begin{array}{l}\text { Theoretical } \\
\text { MW (Da)/pI }\end{array}$ & Function \\
\hline 0118 & TPM1 & $\begin{array}{l}\text { Isoform } 2 \text { of tropomyosin } \alpha-1 \text { chain } \\
\text { Tropomyosin } 1 \alpha \text { variant } 6\end{array}$ & $\begin{array}{l}\text { IPI00745267 } \\
\text { IPI00384369 }\end{array}$ & $3.24 \uparrow^{b}$ & $\begin{array}{l}26664 / 4.77 \\
28720 / 4.75\end{array}$ & Cytoskeletal proteins \\
\hline 0308 & RCN1 & Reticulocalbin-1 precursor & IPI00015842 & $4.20 \uparrow$ & $38866 / 4.86$ & Calcium binding \\
\hline 0309 & RCN1 & Reticulocalbin-1 precursor & IPI00015842 & $3.32 \uparrow$ & $38866 / 4.86$ & Calcium binding \\
\hline 1234 & RPSA & $\begin{array}{l}40 \mathrm{~S} \text { ribosomal protein SA } \\
\text { Ribosomal protein SA }\end{array}$ & $\begin{array}{l}\text { IPI00553164 } \\
\text { IPI00413108 }\end{array}$ & $2.90 \downarrow^{b}$ & $\begin{array}{l}32833 / 4.79 \\
33293 / 4.79\end{array}$ & Protein biosynthesis \\
\hline 1321 & VIM & Vimentin & IPI00418471 & $4.64 \uparrow$ & $53619 / 5.06$ & Cytoskeletal proteins \\
\hline 2117 & SFRS1 & $\begin{array}{l}\text { Isoform ASF-1 of splicing factor, } \\
\text { arginine/serine-rich } 1 \\
\text { Isoform ASF- } 3 \text { of splicing factor, } \\
\text { arginine/serine-rich } 1\end{array}$ & IPI00218592 & $9.73 \uparrow$ & $22446 / 7.72$ & RNA splicing \\
\hline 2217 & SERPINB6 & $\begin{array}{l}\text { Serine (or cysteine) proteinase inhibitor, } \\
\text { clade B (ovalbumin), member } 6\end{array}$ & IPI00749398 & $4.15 \downarrow$ & $42594 / 5.18$ & Protein degradation \\
\hline 2228 & EIF2S1 & $\begin{array}{l}\text { Eukaryotic translation initiation } \\
\text { factor } 2 \text { subunit } 1\end{array}$ & IPI00219678 & $3.25 \uparrow$ & $36089 / 5.02$ & Protein biosynthesis \\
\hline 2338 & TXNDC4 & $\begin{array}{l}\text { Thioredoxin domain-containing } \\
\text { protein } 4 \text { precursor }\end{array}$ & IPI00401264 & $2.74 \uparrow$ & $46941 / 5.09$ & $\begin{array}{l}\text { Cell redox } \\
\text { homeostasis/chaperone }\end{array}$ \\
\hline 3002 & S100A4 & Protein S100-A4 & IPI00032313 & $4.57 \downarrow$ & $11721 / 5.85$ & Calcium binding \\
\hline 3110 & STARD3 & $\begin{array}{l}\text { START domain-containing protein } 3 \\
35 \mathrm{kDa} \text { protein }\end{array}$ & IPI00791229 & $2.83 \uparrow$ & $35037 / 6.04$ & Metabolism \\
\hline 3117 & PSPH & Phosphoserine phosphatase & IPI00019178 & $2.43 \downarrow$ & $24992 / 5.53$ & Metabolism \\
\hline 3218 & TUBB2C & $\begin{array}{l}\text { Tubulin } \beta \text { chain } \\
\text { Tubulin, } \beta \text { polypeptide } \\
\text { Tubulin } \beta-2 C \text { chain }\end{array}$ & $\begin{array}{l}\text { IPI00011654 } \\
\text { IPI00645452 } \\
\text { IPI00007752 }\end{array}$ & $184.06 \uparrow$ & $\begin{array}{l}49639 / 4.78 \\
47736 / 4.70 \\
49799 / 4.79\end{array}$ & Cytoskeletal proteins \\
\hline 3325 & POLD2 & DNA polymerase subunit $\delta-2$ & IPI00025616 & $2.19 \uparrow$ & $51257 / 5.35$ & $\begin{array}{l}\text { DNA replication and } \\
\text { repair }\end{array}$ \\
\hline 3415 & PDIA3 & $\begin{array}{l}\text { Protein disulfide-isomerase A3 } \\
\text { precursor }\end{array}$ & IPI00025252 & $2.09 \uparrow$ & $56747 / 5.98$ & Cell redox homeostasis \\
\hline 3533 & KRT1 & Keratin, type II cytoskeletal 1 & IPI00220327 & $2.48 \uparrow$ & $65978 / 8.16$ & Cytoskeletal proteins \\
\hline 4005 & ABHD14B & $\begin{array}{l}\text { ABHD14B } 22 \mathrm{kDa} \text { protein } \\
\text { Isoform } 1 \text { of abhydrolase } \\
\text { domain-containing protein 14B }\end{array}$ & $\begin{array}{l}\text { IPI00747859 } \\
\text { IPI00063827 }\end{array}$ & $2.17 \uparrow$ & $\begin{array}{l}22367 / 6.04 \\
22332 / 5.94\end{array}$ & Metabolism \\
\hline 4007 & UBE2N & $\begin{array}{l}\text { ubiquitin-conjugating enzyme E2N } \\
16 \mathrm{kDa} \text { protein }\end{array}$ & IPI00874051 & $2.26 \downarrow$ & $16233 / 5.41$ & DNA repair \\
\hline 4010 & S100A11 & Protein S100-A11 & IPI00013895 & $3.75 \downarrow$ & $11733 / 6.56$ & Calcium binding \\
\hline 4118 & DNAJC9 & DNAJ homolog subfamily $\mathrm{C}$ member 9 & IPI00154975 & $2.91 \uparrow$ & $29891 / 5.58$ & Chaperone \\
\hline 4213 & SERPINB1 & Leukocyte elastase inhibitor & IPI00027444 & $2.32 \uparrow$ & $42715 / 5.26$ & Protein degradation \\
\hline 5003 & FABP3 & Fatty acid-binding protein, heart & IPI00219684 & $2.95 \downarrow$ & $14849 / 6.29$ & Metabolism \\
\hline 5020 & ACP1 & $\begin{array}{l}\text { Isoform } 1 \text { of low molecular weight } \\
\text { phosphotyrosine protein phosphatase }\end{array}$ & IPI00219861 & $2.27 \downarrow$ & $18031 / 7.63$ & Metabolism \\
\hline 5204 & DNAJB11 & $\begin{array}{l}\text { DnaJ homolog subfamily B } \\
\text { member } 11 \text { precursor }\end{array}$ & IPI00008454 & $2.38 \uparrow$ & $40489 / 5.81$ & Chaperone \\
\hline
\end{tabular}


Table II. Continued.

\begin{tabular}{|c|c|c|c|c|c|c|}
\hline $\begin{array}{l}\text { SSP } \\
\text { No. }\end{array}$ & Gene symbol & Protein name & Accession No. ${ }^{a}$ & $\begin{array}{c}\text { Fold } \\
\text { change }^{b}\end{array}$ & $\begin{array}{l}\text { Theoretical } \\
\text { MW (Da)/pI }\end{array}$ & Function \\
\hline \multirow[t]{2}{*}{5313} & ENO1 & $\alpha$-enolase & IPI00465248 & $2.56 \downarrow$ & $47139 / 7.01$ & $\begin{array}{l}\text { Metabolism/nucleotide } \\
\text { binding }\end{array}$ \\
\hline & ENO3 & $\beta$-enolase & IPI00218474 & & $46957 / 7.59$ & Metabolism \\
\hline \multirow[t]{3}{*}{5530} & TXNRD1 & TXNRD1 $55 \mathrm{kDa}$ protein & IPI00871867 & $2.04 \downarrow$ & $54512 / 6.07$ & Cell redox homeostasis \\
\hline & & $\begin{array}{l}\text { Thioredoxin reductase } 1 \\
\text { cytoplasmic precursor }\end{array}$ & IPI00554786 & & $54672 / 6.07$ & \\
\hline & & Thioredoxin reductase 1 isoform 1 & IPI00847482 & & $60627 / 6.42$ & \\
\hline \multirow[t]{2}{*}{6115} & PSMD9 & $\begin{array}{l}\text { Isoform p } 27-\mathrm{L} \text { of } 26 \mathrm{~S} \text { proteasome } \\
\text { non-ATPase regulatory subunit } 9\end{array}$ & IPI00010860 & $3.71 \downarrow$ & $24639 / 6.46$ & Protein degration \\
\hline & & $\begin{array}{l}\text { Isoform p27-S of } 26 \mathrm{~S} \text { proteasome } \\
\text { non-ATPase regulatory subunit } 9\end{array}$ & IPI00216220 & & $22722 / 5.38$ & \\
\hline 6205 & ANXA1 & Annexin A1 & IPI00218918 & $2.03 \downarrow$ & $38690 / 6.57$ & Calcium binding \\
\hline 6209 & ACAT2 & Acetyl-CoA acetyltransferase, cytosolic & IPI00291419 & $2.14 \downarrow$ & $41324 / 6.47$ & Metabolism \\
\hline 6305 & CTH & $\begin{array}{l}\text { Isoform } 1 \text { of cystathionine } \gamma \text {-lyase } \\
\text { Isoform } 2 \text { of cystathionine } \gamma \text {-lyase }\end{array}$ & $\begin{array}{l}\text { IPI00031557 } \\
\text { IPI00221301 }\end{array}$ & $2.96 \uparrow$ & $\begin{array}{l}44480 / 6.21 \\
39480 / 6.43\end{array}$ & Metabolism \\
\hline 7015 & EIF1B & $\begin{array}{l}\text { Eukaryotic translation } \\
\text { initiation factor } 1 \mathrm{~b}\end{array}$ & IPI00031489 & $9.23 \downarrow$ & $12816 / 6.82$ & Protein biosynthesis \\
\hline 7117 & ISOC1 & $\begin{array}{l}\text { Isochorismatase domain-containing } \\
\text { protein } 1\end{array}$ & IPI00304082 & $2.54 \downarrow$ & $32216 / 6.96$ & Unkonwn \\
\hline 7118 & PROSC & $\begin{array}{l}\text { Proline synthetase co-transcribed } \\
\text { bacterial homolog protein }\end{array}$ & IPI00016346 & $2.39 \downarrow$ & $30325 / 7.09$ & Unknown \\
\hline \multirow[t]{2}{*}{7122} & GAPDH & $\begin{array}{l}\text { GAPDH } 32 \mathrm{kDa} \text { protein } \\
\text { Glyceraldehyde-3-phosphate } \\
\text { dehydrogenase }\end{array}$ & $\begin{array}{l}\text { IPI00795257 } \\
\text { IPI00219018 }\end{array}$ & $4.36 \uparrow$ & $\begin{array}{l}31528 / 7.15 \\
36030 / 8.57\end{array}$ & Metabolism \\
\hline & & $\begin{array}{l}\text { Glyceraldehyde 3-phosphate } \\
\text { dehydrogenase }\end{array}$ & IPI00789134 & & $27853 / 6.45$ & \\
\hline \multirow[t]{3}{*}{7210} & РCBP2 & PCBP2 protein & IPI00470509 & $2.09 \downarrow$ & $35324 / 8.17$ & Nucleotide binding \\
\hline & & Poly(rC)-binding protein 2 & IPI00216689 & & $38556 / 8.17$ & \\
\hline & PCBP3 & Isoform 4 of Poly(rC)-binding protein 3 & IPI00410587 & & $35788 / 8.22$ & Nucleotide binding \\
\hline \multirow[t]{2}{*}{7626} & FUBP1 & $\begin{array}{l}\text { Isoform } 2 \text { of far upstream } \\
\text { element-binding protein } 1\end{array}$ & IPI00853059 & $2.35 \downarrow$ & $68691 / 6.62$ & Nucleotide binding \\
\hline & & FUBP1 68 kDa protein & IPI00873767 & & $67648 / 6.85$ & \\
\hline 8006 & PFN1 & Profilin-1 & IPI00216691 & $2.67 \downarrow$ & $15045 / 8.44$ & Cytoskeletal proteins \\
\hline \multirow[t]{2}{*}{8023} & CNBP & $\begin{array}{l}\text { Cellular nucleic acid-binding protein } \beta \\
\text { variant } 2\end{array}$ & IPI00430813 & $8.51 \downarrow$ & $18957 / 7.76$ & Nucleotide binding \\
\hline & & $\mathrm{CNBP}$ zinc finger protein & IPI00430812 & & $19579 / 7.76$ & \\
\hline 8040 & $\begin{array}{l}\text { UBE2M } \\
\text { UBE2MP1 }\end{array}$ & $\begin{array}{l}\text { NEDD8-conjugating enzyme Ubc12 } \\
\text { Similar to NEDD8-conjugating } \\
\text { enzyme Ubc12 }\end{array}$ & $\begin{array}{l}\text { IPI00022597 } \\
\text { IPI00457179 }\end{array}$ & $2.83 \downarrow$ & $\begin{array}{l}20887 / 7.57 \\
19091 / 7.55\end{array}$ & Protein degradation \\
\hline \multirow[t]{3}{*}{8103} & VDAC2 & $\begin{array}{l}\text { Isoform } 3 \text { of voltage-dependent } \\
\text { anion-selective channel protein } 2\end{array}$ & IPI00216026 & $2.08 \downarrow$ & $31547 / 7.49$ & $\begin{array}{l}\text { Transmembrane } \\
\text { proteins }\end{array}$ \\
\hline & & VDAC2 $30 \mathrm{kDa}$ protein & IPI00855744 & & $30329 / 8.00$ & \\
\hline & & $\begin{array}{l}\text { Isoform } 2 \text { of voltage-dependent } \\
\text { anion-selective channel protein } 2\end{array}$ & IPI00216024 & & $30393 / 6.81$ & \\
\hline 8110 & EIF4H & $\begin{array}{l}\text { Isoform long of eukaryotic translation } \\
\text { initiation factor } 4 \mathrm{H}\end{array}$ & IPI00014263 & $3.44 \downarrow$ & $27368 / 6.67$ & Protein biosynthesis \\
\hline
\end{tabular}


Table II. Continued.

\begin{tabular}{|c|c|c|c|c|c|c|}
\hline $\begin{array}{l}\text { SSP } \\
\text { No. }\end{array}$ & Gene symbol & Protein name & Accession No. ${ }^{a}$ & $\begin{array}{c}\text { Fold } \\
\text { change }^{b}\end{array}$ & $\begin{array}{l}\text { Theoretical } \\
\text { MW (Da)/pI }\end{array}$ & Function \\
\hline \multirow[t]{3}{*}{8115} & AK2 & $\begin{array}{l}\text { Isoform } 2 \text { of adenylate kinase } \\
\text { isoenzyme } 2 \text {, mitochondrial }\end{array}$ & IPI00218988 & $2.46 \downarrow$ & $25598 / 7.71$ & Metabolism/apoptosis \\
\hline & & $\begin{array}{l}\text { Isoform } 1 \text { of adenylate kinase } \\
\text { isoenzyme } 2 \text {, mitochondrial }\end{array}$ & IPI00215901 & & $26461 / 7.67$ & \\
\hline & & $\begin{array}{l}\text { Isoform } 3 \text { of adenylate kinase } \\
\text { isoenzyme } 2 \text {, mitochondrial }\end{array}$ & IPI00172460 & & $22251 / 8.94$ & \\
\hline 8517 & DAK & Dihydroxyacetone kinase & IPI00551024 & $2.39 \uparrow$ & $58940 / 7.12$ & Metabolism \\
\hline 9003 & SSBP1 & $\begin{array}{l}\text { Single-stranded DNA-binding protein, } \\
\text { mitochondrial precursor }\end{array}$ & IPI00029744 & $2.03 \downarrow$ & $17249 / 9.59$ & DNA repair \\
\hline
\end{tabular}

aIPI_human database accession number. ${ }^{\text {b}}$ Fold changes are represented as mean values calculated from three-repeated experiments. $\uparrow$, spot quantity increased in EC109/CDDP in comparison to EC109; $\downarrow$, spot quantity decreased in EC109/CDDP in comparison to EC109 (Student's t-test, P<0.05).

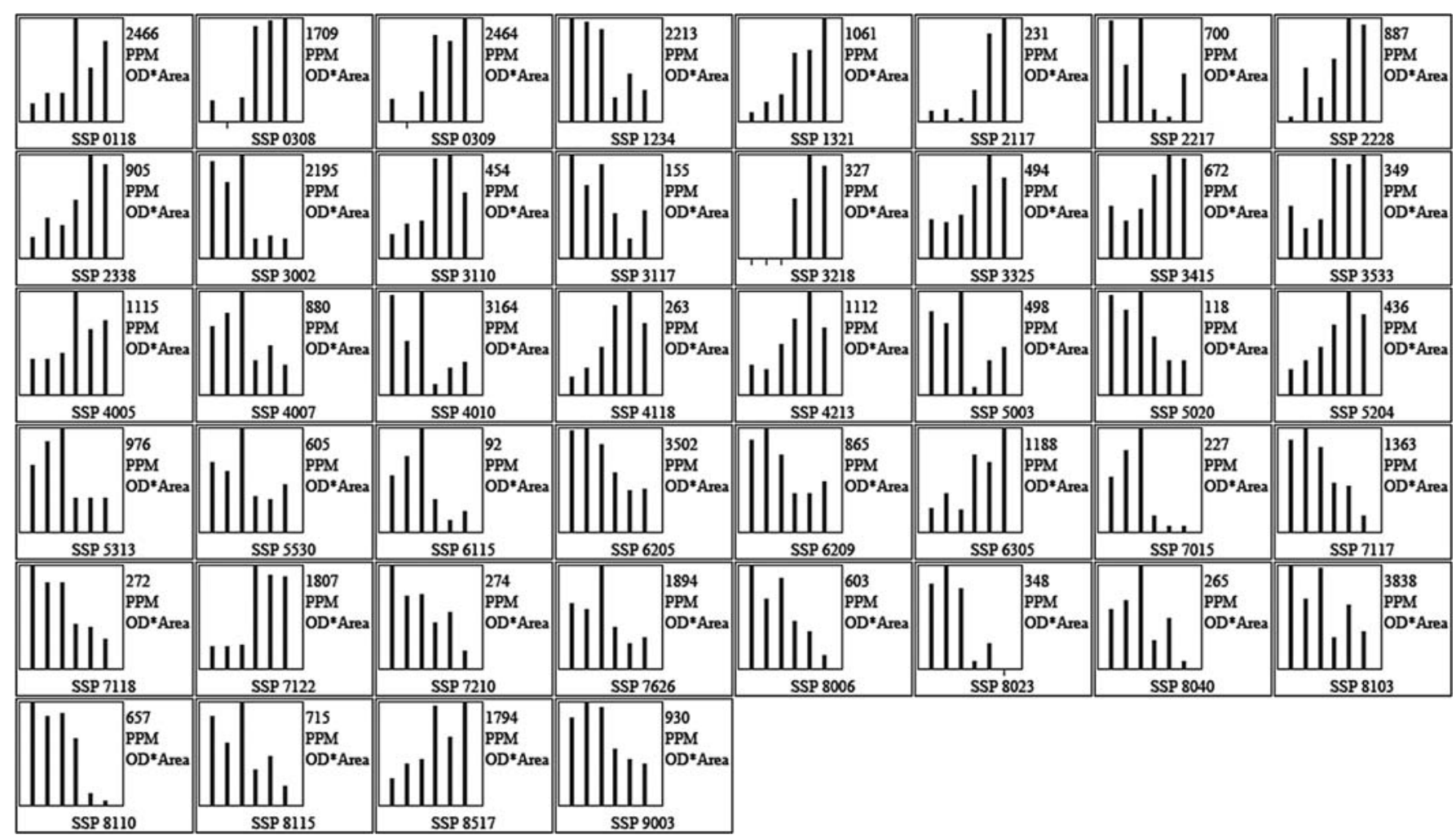

Figure 3. Histogram graphs showing the quantities of 44 differential protein spots between EC109 and EC109/CDDP cells. Bar, protein spot's quantity in each gel. Left three bars, quantities of the protein spots on three separate gels from the parental EC109 cells; right three bars, quantities of the protein spots on three separate gels from the drug-resistant EC109/CDDP cells. SSP, standard spot. The number at the upper right of the histogram is the quantitation of the maximum bar in the graph. The other bars are drawn proportional to the highest bar. PPM (parts per million) is a constant, by which the normalized quantity is multiplied to give a more meaningful value.

Western blot image (Fig. 4A) of CTH showed two distinct bands at approximate 44.5 and $37.5 \mathrm{kDa}$, respectively, identical to the molecular weights of the two isoforms CTH1 and $\mathrm{CTH} 2$. CTH2 protein expression was quite weak, and no marked change was observed between EC109 and EC109/CDDP cells. After normalization to B-actin, the expression levels of TXNDC4 in EC109 and EC109/CDDP cells were $0.1866 \pm 0.04248$ and $0.3970 \pm 0.08417$, respectively, and that of CTH1 was $0.5464 \pm 0.02129$ and $1.288 \pm 0.3903$, respectively. The TXNDC4 and CTH1 protein expression ratio of EC109/CDDP to EC109 cells were 2.929 ( $\mathrm{P}<0.001)$ and $3.244(\mathrm{P}=0.018)$, respectively (Fig. 4B), consistent with the data shown in Fig. 3 and Table II obtained using the proteomic approach. 
A

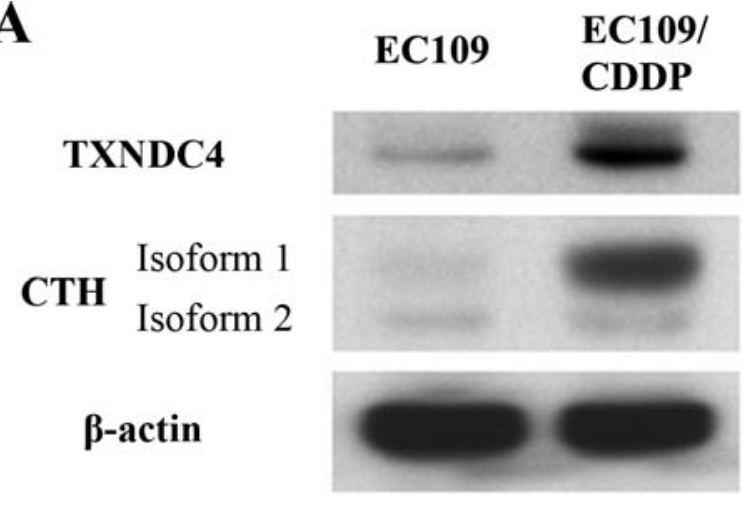

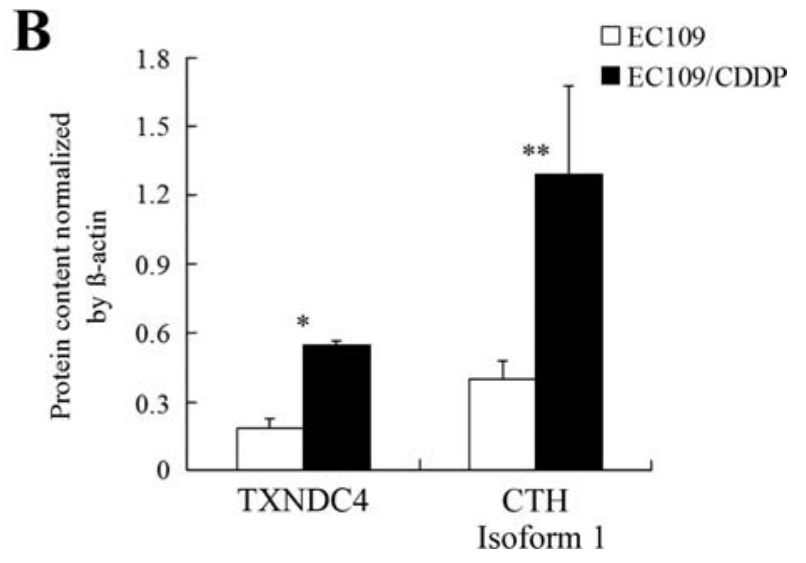

Figure 4. Differential expressed proteins TXNDC4 and CTH identified by proteomic approach were validated by Western blot. (A) Western blot image of TXNDC4, CTH and ß-actin in EC109 and EC109/CDDP cells. (B) Protein expression levels of TXNDC4 and CTH after normalization relative to ß-actin. ${ }^{*} \mathrm{P}<0.001 ;{ }^{* *} \mathrm{P}=0.018$.

A

TXNDC4

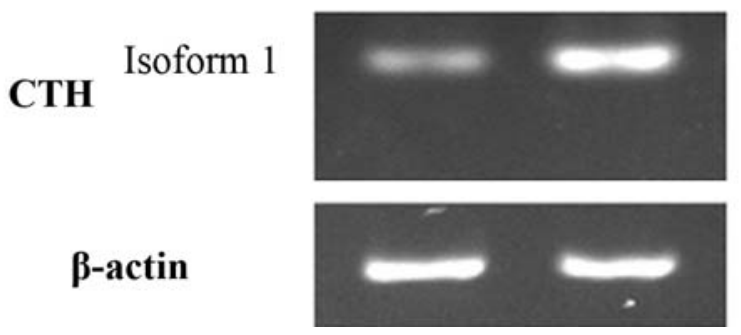

B

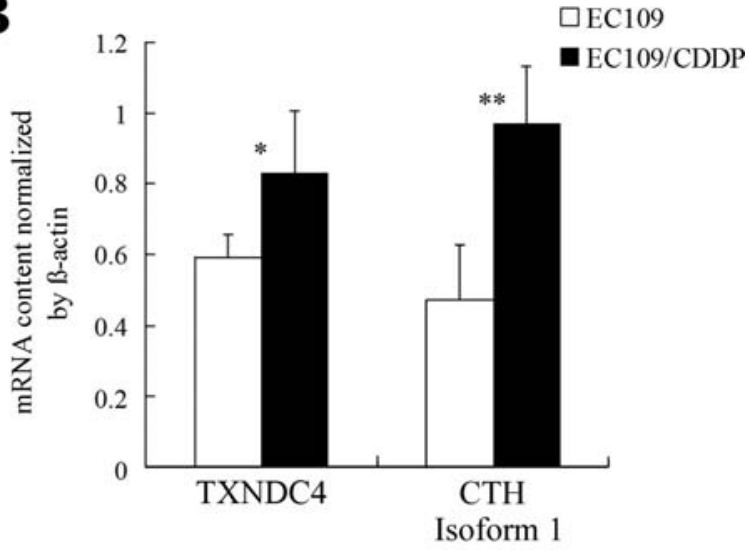

Figure 5. Differential expression of proteins TXNDC4 and CTH identified by proteomic approach were validated by RT-PCR. (A) Agrose electrophoresis image of TXNDC4, CTH and B-actin PCR products in EC109 and EC109/CDDP cells. (B) mRNA expression levels of TXNDC4 and CTH after normalization relative to $\beta$-actin. ${ }^{*} \mathrm{P}=0.094 ;{ }^{* *} \mathrm{P}=0.018$.

Semi-quantative RT-PCR. The mRNA expression of TXNDC4 and CTH in EC109 and EC109/CDDP cells were determined using semi-quantitative RT-PCR. Gene expression profile of TXNDC4 and CTH is shown in Fig. 5A. For CTH, only one band at approximate 800 bp was visible on the gel, which was CTH Isoform 1 PCR product. No CTH Isoform 2 PCR product at 668 bp could be detected in either EC109 or EC109/CDDP cells. After normalized to B-actin, the expression level of TXNDC4 in EC109 and EC109/CDDP cells was $0.5902 \pm 0.06689$ and $0.8297 \pm 0.1778$, respectively, and that of CTH1 was $0.4732 \pm 0.1504$ and $0.9699 \pm 0.1643$, respectively. The TXNDC4 and CTH1 mRNA expression ratio of EC109/CDDP to EC109 cells was $1.406(\mathrm{P}=0.094)$ and $2.050(\mathrm{P}=0.018)$, respectively (Fig. 5B). The mRNA levels of TXNDC4 and CTH1 in EC109 and EC109/CDDP cells were in accordance with the protein levels based on both 2-DE and Western blot analyses.

\section{Discussion}

ESCC patients generally show different degrees of chemosensitivity, even those with the same disease stage and thera- peutic regimen. Drug-resistance acts as a barrier in esophageal cancer therapy, and it affects the prognosis of patients in neoadjuvant and adjuvant settings $(17,18)$. Therefore, it is important to elucidate the mechanisms of drug resistance in order to improve the prognosis of esophageal cancer patients.

In our previous work, we established a drug-resistant cell line, EC109/CDDP cells, by pulse treatment of the most widely-used antitumor drug, cisplatin to the ESCC EC109 cells, and carried out experiments intending to find out the potential molecular mechanisms involved in ESCC drugresistant phenotype. However, the well-known multidrug resistance-related genes, ATP-binding cassette, sub-family B, member 1 (MDR1), ATP-binding cassette, sub-family C, member 1 (MRP1), ATP-binding cassette, sub-family G, member 2 (ABCG2), lung resistance protein (LRP), and glutathione S-transferase $\pi$ (GST- $\pi$ ) might not completely explain its multi-drug resistance (14). We, therefore, used high throughput proteomics approach to investigate the protein expression change of EC109/CDDP, so as to help understanding of the molecular mechanism of ESCC drug resistance and to identify candidate biomarkers of anticancer drug response or novel therapeutic targets. 
Forty-four proteins exhibited at least 2-fold changes of expression level in the multidrug-resistant cell line EC109/ CDDP compared with its counterpart EC109 identified by MS analysis. To confirm the proteomic results, two proteins CTH and TXNDC4, in which we are interested, were selected and analyzed by Western blot and RT-PCR. The changes of these two proteins and genes between EC109 and EC109/CDDP cells by Western blot and RT-PCR were in accordance with the 2-DE results, confirming the veracity of the 2-DE gel image data and providing a rationale for future functional studies of these proteins.

An investigation of the reported functions of each individual identified protein was made. According to the available information in the Universal Protein Resource (UniProt) and published literatures, these proteins could be divided into several major groups: endoplasmic reticulum (ER) proteins in response to stress, metabolic enzymes, proteins involved in DNA replication and repair, nucleotide binding proteins, calcium binding proteins, cytoskeletal proteins, etc (Table II).

Four proteins, TXNDC4 (SSP 2338), protein disulfideisomerase A3 precursor (PDIA3, SSP 3415), DnaJ homolog subfamily C member 9 (DNAJC9, SSP 4118) and DnaJ homolog subfamily B member 11 precursor (DNAJB11, SSP 5204), located in the ER and involved in the ER stress response, were up-regulated in EC109/CDDP cells. Cisplatin was reported to induce ER stress (19), therefore, we assume that EC109/CDDP cells were under ER stress induced by cisplatin. TXNDC4 and PDIA3 are members of the thioredoxin protein family, which assists in oxidative protein folding so as to relieve the overloading of mis- or unfolded proteins $(20,21)$. TXNDC4 and PDIA3 function in controlling the ER and cytosolic $\mathrm{Ca}^{2+}$ homeostasis $(22,23)$, thus keeping the cells away from apoptosis brought about by the cellular $\mathrm{Ca}^{2+}$ overload or perturbation of intracellular $\mathrm{Ca}^{2+}$ compartmentalization (24). Up-regulation of TXNDC4 and PDIA3 in EC109/CDDP might confer the cell resistance to apoptosis induced by cytotoxic drugs through their modulation of both the ER stress and $\mathrm{Ca}^{2+}$ homeostasis. DNAJC9 and DNAJB11 are members of DNAJ/HSP40s family, which plays important roles in regulating cell proliferation, survival and apoptosis by serving as chaperones for HSP70s (25). The induction of HSP70 members under stress conditions is a major UPR protective response and has been best characterized for its role in contributing to resistance to a wide variety of chemotherapeutic agents (including taxol, docetaxol, cisplatin, etoposide and celetoxib) in multiple tumor types $(26,27)$. DNAJB11 and DNAJC9 might play an indirect role in drug resistance through their regulation of HSP70s.

According to previous studies, ER stress synergizes with cisplatin to more efficiently kill tumor cells, although it induces protection against topoisomerase-II-targeted chemotherapeutic agents (28). This is partly contrary to the fact that EC109/CDDP cells showed resistance to both cisplatin and etoposide at the same time. Thus, we are not sure whether the ER stress could account for EC109/CDDP cells' multi-drug resistant phenotype or just some of the drugs tested in our previous work (14). A more comprehensive understanding of which drugs the ER stress alters sensitivity to is essential, and EC109/CDDP would serve as an important cell model in research on the relationship between ER stress and cancer drug resistance.

A large group of metabolic enzymes were found to express differentially between EC109/CDDP and EC109 cells. SSP 6305 up-regulated in EC109/CDDP 2-DE gels was identified as protein CTH1 and/or CTH2 by MS analysis. $\mathrm{CTH}$ is an enzyme that catalyzes the conversion of cystathionine into cysteine, which was further metabolized to yield glutathione $(\mathrm{GSH})$ and metallothionein (MT). Previous studies have shown that repeated exposure to cisplatin induced MT and GSH in vivo and in vitro and produced resistance to cisplatin by metabolizing it to an inactive form (29). Furthermore, inhibiting CTH enzymatic activity could restore the antitumor activity of cisplatin (30). Alternative splicing of this gene results in two transcript variants encoding different protein isoforms CTH1 and CTH2. Levonen et al (31) found that CTH1 was the predominant variant in human cells, and only cells over-expressing CTH1 rather than CTH2 had increased activity. In our work, Western blot showed it was the up-regulation of CTH1, but not CTH2 that plays a part in EC109/CDDP cell drug-resistance.

Besides CTH, other enzymes identified in this experiment include the up-regulation of START domain-containing protein 3 (SSP3110), abhydrolase domain-containing protein 14B (SSP4005) and dihydroxyacetone kinase (SSP8517), and down-regulation of acetyl-CoA acetyltransferase and fatty acid-binding protein (SSP6209), phosphoserine phosphatase (SSP3117), isoform 1 of low molecular weight phosphotyrosine protein phosphatase (SSP5020) and adenylate kinase isoenzyme 2 (SSP8115) in EC109/CDDP cells. The differential expression of these enzymes between EC109 and EC109/CDDP cells might reflect the change of EC109/ CDDP in metabolism to survive chronic environmental stresses, such as the drug attack.

Moreover, several proteins involved in DNA replication and repair (DNA polymerase subunit $\delta-2$, single-stranded DNA-binding protein mitochondrial precursor and uniquitinconjugating enzyme E2N), protein biosyntheses and degradation (ribosomal protein SA, eukaryotic translation initiation factor 2 subunit 1, eukaryotic translation initiation factor $1 \mathrm{~b}$, isoform long of eukaryotic translation initiation factor $4 \mathrm{H}, 26 \mathrm{~S}$ proteasome non-ATPase regulatory subunit 9 and NEDD8-conjugating enzyme Ubc12), nucleotide binding [poly(rC)-binding protein, far upstream element-binding protein $1, \alpha$-enolase, splicing factor arginine/ serine-rich 1 and cellular nucleic acid binding protein], calcium binding (RCN1, annexin A1, protein S100-A4 and S100-A11), cytoskeletal proteins (tubulin $B$ chain, tropomyosin $\alpha-1$ chain, profilin-1, vimentin and keratin 1) and a transmembrane protein (voltage dependent anion selective channel 2) have been identified to express differentially between EC109/ CDDP and EC109 cells. Some of them have been identified by previous 2-DE comparative proteomic analyses in various drug-resistant cancer cell lines (13). However, their participation in cancer drug resistance has not been discussed or studied extensively. Thus, we cannot make any assumption or conclusion regarding their possible roles in EC109 and EC109/CDDP cells.

Although we have identified scores of proteins with potential involvement in EC109/CDDP drug resistance, it has 
to be admitted that the comparative proteomic approach based on 2-DE has some inherent technical limitations, for example, the deficiency in detection of proteins with lower abundance, the separation and identification of hydrophobic membrane proteins and proteins with extreme mass or $\mathrm{pI}$. Furthermore, most of the proteins identified in the proteomic analyses have not been validated for their expression changes using another approach such as Western blot and for their role in drug resistance using functional assays. In future studies, validation and function analysis of the results originated from proteomic researches are not only desirable, but essential.

In conclusion, we identified candidate proteins involved in multiple biological functions that were differentially expressed in the multi-drug resistant cell line EC109/CDDP compared to parental cell line EC109 by comparative proteomic approach based on 2-DE. Some of the identified proteins in our work have not been reported to be related to cancer drug resistance before, such as TXNDC4, and may motivate us to look into ESCC drug resistance in a fresh perspective and generate novel hypotheses in addition to the well-known mechanisms. Further functional analysis in vitro and validation in clinical ESCC samples of these putative proteins to make certain their roles and mechanism in ESCC chemotherapeutic resistance are necessary and underway.

\section{Acknowledgements}

The study was supported by Guangdong Natural Science Foundation (Grants No. 7001531).

\section{References}

1. Parkin DM, Bray FI and Devesa SS: Cancer burden in the year 2000. The global picture. Eur J Cancer 37 (Suppl 8): S4-S66, 2001.

2. Siewert JR and Ott K: Are squamous and adenocarcinomas of the esophagus the same disease? Semin Radiat Oncol 17: 38-44, 2007.

3. Stein HJ, Sendler A, Fink U and Siewert JR: Multidisciplinary approach to esophageal and gastric cancer. Surg Clin North Am 80: 659-682, 2000.

4. Gebski V, Burmeister B, Smithers BM, Foo K, Zalcberg J, Simes J and Australasian Gastro-Intestinal Trials G: Survival benefits from neoadjuvant chemoradiotherapy or chemotherapy in oesophageal carcinoma: a meta-analysis. Lancet Oncol 8: 226-234, 2007.

5. Izzo JG, Malhotra U, Wu TT, et al: Association of activated transcription factor nuclear factor $\kappa \mathrm{B}$ with chemoradiation resistance and poor outcome in esophageal carcinoma. J Clin Oncol 24: 748-754, 2006.

6. Vallbohmer D and Lenz HJ: Predictive and prognostic molecular markers in outcome of esophageal cancer. Dis Esophagus 19: 425-432, 2006.

7. Tanaka E, Hashimoto Y, Ito T, et al: The suppression of auroraA/STK15/BTAK expression enhances chemosensitivity to docetaxel in human esophageal squamous cell carcinoma. Clin Cancer Res 13: 1331-1340, 2007.

8. Nam TK, Lee JH, Cho SH, et al: Low hMLH1 expression prior to definitive chemoradiotherapy predicts poor prognosis in esophageal squamous cell carcinoma. Cancer Lett 260: 109-117, 2008.

9. Zhang S, Ding F, Luo A, et al: XIAP is highly expressed in esophageal cancer and its downregulation by RNAi sensitizes esophageal carcinoma cell lines to chemotherapeutics. Cancer Biol Ther 6: 973-980, 2007.
10. Wang H, Qian H, Yu J, et al: Administration of PUMA adenovirus increases the sensitivity of esophageal cancer cells to anticancer drugs. Cancer Biol Ther 5: 380-385, 2006.

11. Liu TL, Shimada H, Ochiai T, et al: Enhancement of chemosensitivity toward peplomycin by calpastatin-stabilized NF-кB p65 in esophageal carcinoma cells: possible involvement of Fas/Fas-L synergism. Apoptosis 11: 1025-1037, 2006.

12. Toshimitsu H, Hashimoto K, Tangoku A, et al: Molecular signature linked to acquired resistance to cisplatin in esophageal cancer cells. Cancer Lett 211: 69-78, 2004.

13. Zhang JT and Liu Y: Use of comparative proteomics to identify potential resistance mechanisms in cancer treatment. Cancer Treat Rev 33: 741-756, 2007.

14. Wen J, Zheng B, Hu Y, et al: Establishment and biological analysis of the EC109/CDDP multidrug-resistant esophageal squamous cell carcinoma cell line. Oncol Rep 22: 65-71, 2009.

15. Fu L, Qin YR, Xie D, et al: Identification of alpha-actinin 4 and $67 \mathrm{kDa}$ laminin receptor as stage-specific markers in esophageal cancer via proteomic approaches. Cancer 110: 2672-2681, 2007.

16. Molloy MP, Brzezinski EE, Hang J, McDowell MT and VanBogelen RA: Overcoming technical variation and biological variation in quantitative proteomics. Proteomics 3: 1912-1919, 2003.

17. Berger AC, Farma J, Scott WJ, et al: Complete response to neoadjuvant chemoradiotherapy in esophageal carcinoma is associated with significantly improved survival. J Clin Oncol 23: 4330-4337, 2005.

18. Kelsen DP, Winter KA, Gunderson LL, et al: Long-term results of RTOG trial 8911 (USA Intergroup 113): a random assignment trial comparison of chemotherapy followed by surgery compared with surgery alone for esophageal cancer. J Clin Oncol 25: 3719-3725, 2007.

19. Mandic A, Hansson J, Linder S and Shoshan MC: Cisplatin induces endoplasmic reticulum stress and nucleus-independent apoptotic signaling. J Biol Chem 278: 9100-9106, 2003.

20. Ellgaard L and Frickel EM: Calnexin, calreticulin, and ERp57: teammates in glycoprotein folding. Cell Biochem Biophys 39: 223-247, 2003.

21. Anelli T, Alessio M, Mezghrani A, Simmen T, Talamo F, Bachi A and Sitia R: ERp44, a novel endoplasmic reticulum folding assistant of the thioredoxin family. EMBO J 21: 835-844, 2002.

22. Higo T, Hattori M, Nakamura T, Natsume T, Michikawa T and Mikoshiba K: Subtype-specific and ER lumenal environmentdependent regulation of inositol 1,4,5-trisphosphate receptor type 1 by ERp44. Cell 120: 85-98, 2005.

23. Li Y and Camacho P: $\mathrm{Ca}^{2+}$-dependent redox modulation of SERCA 2b by ERp57. J Cell Biol 164: 35-46, 2004.

24. Orrenius S, Zhivotovsky B and Nicotera P: Regulation of cell death: the calcium-apoptosis link. Nat Rev Mol Cell Biol 4: 552-565, 2003.

25. Qiu XB, Shao YM, Miao S and Wang L: The diversity of the DnaJ/Hsp40 family, the crucial partners for Hsp70 chaperones. Cell Mol Life Sci 63: 2560-2570, 2006.

26. Lee AS: GRP78 induction in cancer: therapeutic and prognostic implications. Cancer Res 67: 3496-3499, 2007.

27. Li J and Lee AS: Stress induction of GRP78/BiP and its role in cancer. Curr Mol Med 6: 45-54, 2006.

28. Ma Y and Hendershot LM: The role of the unfolded protein response in tumour development: friend or foe? Nat Rev Cancer 4: 966-977, 2004.

29. Siddik ZH: Cisplatin: mode of cytotoxic action and molecular basis of resistance. Oncogene 22: 7265-7279, 2003.

30. Saga Y, Hashimoto H, Yachiku S, Iwata T and Tokumitsu M: Reversal of acquired cisplatin resistance by modulation of metallothionein in transplanted murine tumors. Int J Urol 11: 407-415, 2004.

31. Levonen AL, Lapatto R, Saksela M and Raivio KO: Human cystathionine gamma-lyase: developmental and in vitro expression of two isoforms. Biochem J 347: 291-295, 2000. 